\title{
Self-Assembly of Ternary Insulin-Polyethylenimine (PEI)-DNA Nanoparticles for Enhanced Gene Delivery and Expression in Alveolar Epithelial Cells
}

\author{
Markus Elfinger, ${ }^{\dagger, \ddagger, \S}$ Corinna Pfeifer, ${ }^{\dagger, \ddagger, \S}$ Senta Uezguen, ${ }^{\ddagger, \S}$ Monika M. Golas, ${ }^{\perp}$ \\ Bjoern Sander, ${ }^{\perp}$ Christof Maucksch, ${ }^{\ddagger}, \S$ Holger Stark, ${ }^{\perp}$ Manish K. Aneja, ${ }^{\ddagger}$ and \\ Carsten Rudolph ${ }^{\star, \neq, \S}$ \\ Department of Pediatrics, Ludwig-Maximilians University, 80337 Munich, Germany, Department of \\ Pharmacy, Free University of Berlin, 14166 Berlin, Germany, and Three-Dimensional Electron \\ Cryomicroscopy Group, Max Planck Institute for Biophysical Chemistry, 37077 Goettingen, Germany
}

Received June 26, 2009; Revised Manuscript Received August 7, 2009

\begin{abstract}
Enhancing gene delivery and expression in alveolar epithelial cells could offer the opportunity for the treatment of acquired and inherited lung diseases. Here, we show that particle adsorption of human insulin (INS) is capable of increasing plasmid DNA (pDNA) delivery from polyethylenimine (PEI) nanoparticles specifically in alveolar epithelial cells. INS receptors were predominantly detected on alveolar but not on bronchial epithelial cells. INS was adsorbed on the surface of PEI gene vectors by spontaneous self-assembly resulting in ternary PEI-pDNA-INS nanoparticles. Surface adsorption was confirmed by particle size, surface charge, and fluorescence resonance energy transfer (FRET) measurements. INS adsorption significantly increased gene expression of PEI-pDNA nanoparticles up to 16-fold on alveolar epithelial cells but not on bronchial epithelial cells. This increased gene expression was INS receptor specific. Our results demonstrate that targeting INS receptor for gene delivery in alveolar epithelial cells represents a promising approach for enhanced gene delivery and expression.
\end{abstract}

\section{Introduction}

Although gene therapy has not yet been established as a standard therapeutic treatment, it holds potential for the treatment of patients suffering from various acquired and inherited diseases, for which conventional clinical procedures have poor efficacy. Presently, gene medicine is largely limited by the fact that it is not possible to realize the full potential of these new therapeutic approaches until the issue of nucleic acid delivery has been adequately resolved. An ideal gene delivery system should efficiently deliver the transgene into the target cells without inducing immune response or cytotoxicity. Viral vectors offer high transfection efficiencies, but their widespread usage is still limited due to safety concerns, such as immunogenicity and integration into the host genome which may lead to insertional mutagenesis. ${ }^{1}$ Therefore, nonviral systems such as cationic lipids and polymers present themselves as safer alternatives. Nonviral gene vectors composed of plasmid DNA (pDNA) and cationic polymers are promising vector systems which have been extensively investigated for gene delivery. Among cationic polymers, polyethylenimine (PEI) has been shown to have one of the highest transfection efficiencies both in vitro and in vivo and, therefore, has evolved as a "gold standard" in nonviral gene delivery. ${ }^{2,3}$ Its high charge density due to primary, secondary, and tertiary amines allows PEI to bind and compact pDNA into nanoparticles, thereby protecting it from nuclease degradation. Nevertheless, one significant limitation of PEI gene vectors is their lacking capacity to restrict

* To whom correspondence should be addressed. Telephone: (49) 89 5160 7711. Fax: (49) 895160 7846. E-mail: carsten.rudolph@ med.uni-muenchen.de.

These authors contributed equally to this work.

$\doteqdot$ Ludwig-Maximilians University.

${ }^{\S}$ Free University of Berlin.

${ }^{\perp}$ Max Planck Institute for Biophysical Chemistry. transfection to only the desired targeted cells which results from their cellular uptake via ubiquitously expressed heparan sulfate proteoglycans (HSPGs), for example, syndecans. ${ }^{4}$ A high specificity toward target cells is desirable to improve the efficacy of PEI gene vectors and prevent side effects in nontarget tissue.

Receptor-mediated endocytosis may provide a more specific pathway by which PEI gene vectors are taken up into the target cells. Various targeting ligands have been covalently conjugated to PEI, including antibodies, ${ }^{5}$ peptides, ${ }^{6}$ transferrin, ${ }^{7}$ lactoferrin, ${ }^{8}$ sugars, ${ }^{9}$ mannuronic acid, ${ }^{10}$ folate, ${ }^{11}$ and growth factors such as EGF. ${ }^{12}$ However, covalent coupling requires chemical reactions which bear the risk of inactivating the receptor-binding capacity of coupling-sensitive targeting ligands. Therefore, ligand introduction via electrostatic interaction on the surface of the nanoparticles offers an alternative to avoid such limitations.

Insulin (INS) is a peptide hormone composed of 51 amino acid residues and a molecular weight of $5890 \mathrm{Da}$, which is produced by the islets of Langerhans in the pancreas. It regulates carbohydrate, lipid, and protein metabolism and also functions as a growth factor in many cells and tissues. In terms of pharmacotherapy, it is used to treat diabetes mellitus. It has previously been shown that INS receptors are expressed on alveolar epithelial cells. ${ }^{13}$ Once INS is internalized into the cell, it dissociates from its receptor in the endosome and is translocated to the cytoplasm, where most INS is degraded. A minor fraction of the INS translocates further into the cell nucleus. ${ }^{14-16}$ Targeting of insulin receptor in human glioma cells has been shown to result in higher gene expression compared to values observed when either EGF or transferrin receptor was targeted. ${ }^{17}$ It has been proposed that the increased gene expression may be derived from nuclear targeting properties of the insulin receptor system. ${ }^{14,17}$ Though these observations indicate that INS may be a promising candidate as a targeting ligand for 
nonviral gene transfer, gene delivery and expression resulting from targeting the insulin receptor in lung cells remains unexplored.

In the present study, we investigated the self-assembly of ternary nanoparticles consisting of PEI, pDNA, and surfaceadsorbed human INS and characterized their transfection efficiency on lung epithelial cells. In addition, cytotoxicity and biophysical properties of PEI gene vectors coated with increasing amounts of insulin were evaluated.

\section{Experimental Section}

Chemicals and Plasmids. Human insulin (\#19278), branched polyethylenimine (PEI, average molecular weight $25 \mathrm{kDa}$ ), insulinFITC labeled from bovine pancreas (INS-FITC), and 5(6)-carboxy-Xrhodamine $N$-succinimidyl ester (X-rhodamine) were purchased from Sigma-Aldrich (Schnelldorf, Germany) and used without further purification. PEI was diluted in double-distilled water and adjusted to $\mathrm{pH} 7.4$ with $\mathrm{HCl}$. INS-FITC was diluted in double-distilled water to a final concentration of $1 \mathrm{mg} / \mathrm{mL}$. Purified mouse antihuman CD220 antibody (monoclonal; clone 3B6/IR) was purchased from BD Pharmingen (Heidelberg, Germany). Alexa Fluor 488 labeled rabbit antimouse IgG was obtained from Molecular Probes (Leiden, The Netherlands). The plasmid pCMVLuc containing the Photinus pyralis luciferase gene under the control of the cytomegalovirus immediate early promoter (CMV) was kindly provided by Prof. E. Wagner (Department of Pharmacy, Ludwig Maximilians University, Munich, Germany). The pEGFP-N1 plasmid contains one copy of EGFP cDNA driven by the CMV promoter and was obtained from Clontech (Clontech, USA). Both plasmids were propagated in Escherichia coli and provided in a highly purified form (LPS content $\leq 0.1$ E.U./ $\mu \mathrm{g}$ DNA) by PlasmidFactory GmbH \& Co. KG (Bielefeld, Germany). The amount of supercoiled pDNA was $\geq 90 \%$ ccc (covalently closed circular) grade.

Cell Lines. A549 (human alveolar-like epithelial) cells were obtained from the DSMZ (German Collection of Microorganisms and Cell Cultures, Braunschweig, Germany) and BEAS-2B (human bronchial epithelial) cells were purchased from the ATCC (American type Culture Collection). Both cell lines were grown in minimum essential medium (MEM, Gibco-BRL, Karlsruhe, Germany), supplemented with $10 \%$ fetal calf serum (FCS, Gibco-BRL) at $37{ }^{\circ} \mathrm{C}$ in a $5 \% \mathrm{CO}_{2}$ humidified air atmosphere.

Investigation of the INS Receptor. INS receptor status on A549 and BEAS-2B cells was investigated using fluorescence-activated cell sorting (FACS) and fluorescence microscopy after antibody staining. For FACS measurement experiments, 100000 cells per well were seeded in 24-well plates (TPP, Trasadingen, Switzerland) $24 \mathrm{~h}$ prior to antibody incubation. Fluorescence microscopy experiments were arranged in 4-chamber slides (NUNC, Rochester, NY) with 25000 cells per chamber. Mouse antihuman CD220 antibody was diluted 1:200 in ice-cold phosphate buffered saline (PBS) and incubated on the cells for $30 \mathrm{~min}$ at $0{ }^{\circ} \mathrm{C}$. After washing the cells three times with PBS, a 1:50 dilution of the secondary fluorescence-labeled antibody was added to the cells on ice for another $30 \mathrm{~min}$. To analyze unspecific binding, control experiments were performed using only the secondary antibody. Cells were washed three times with PBS and scraped off into $500 \mu \mathrm{L}$ of PBS. FACS measurements were performed using a Becton Dickinson FACScan (San Jose, CA). For fluorescence microscopy experiments, cells were rinsed and fixed in $4 \%$ para-formaldehyde, followed by staining of cell nuclei with $0.33 \mu \mathrm{M} \mathrm{4}$ ',6-diamidino-2-phenylindole (DAPI) for $3 \mathrm{~min}$. The slides were covered with mounting medium (Vectashield, Vector Laboratories Inc., Burlingame, CA), and microscopy was done using an epifluorescence Axiovert 135 microscope (Carl Zeiss, Jena, Germany).

Preparation of Gene Vector Complexes. Plasmid DNA (pCMVLuc or pEGFP-N1) and PEI were diluted separately in $25 \mu \mathrm{L}$ of doubledistilled water with an N/P ratio (molar ratio of PEI nitrogen to DNA phosphate) of 8 . The pDNA solution was added to the polymer solution

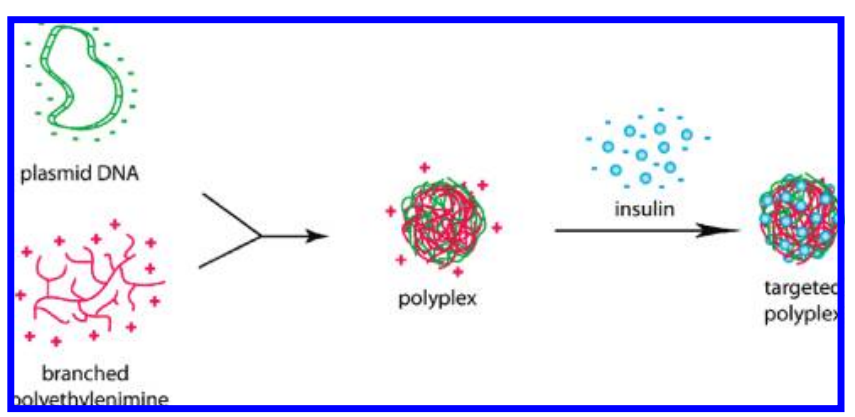

Figure 1. Complexation scheme of ternary PEI-pDNA-INS polyplexes. Plasmid DNA was complexed for 20 min with polyethylenimine to result in PEI-pDNA complexes. Subsequently, human INS was added and incubated for another $30 \mathrm{~min}$, which resulted in the adsorption of INS on the particle surface.

and gently mixed by pipetting up and down 5-8 times. Polyplexes with a concentration of $20 \mu \mathrm{g}$ pDNA/mL were incubated for $20 \mathrm{~min}$ at room temperature. To form ternary-coated complexes, increasing amounts of human INS ranging from 1 to $10 \mu \mathrm{g}$ INS per $\mu \mathrm{g}$ of pDNA were added to the solutions and incubated for another $30 \mathrm{~min}$. An overview of the complex preparation scheme is given in Figure 1.

Size and Zeta Potential Measurements. Particle size (determined by dynamic light scattering) and the zeta potential were measured using a ZetaPALS/Zeta Potential Analyzer (Brookhaven Instruments Corporation, Vienna, Austria). Gene vector complexes were generated as described above with pCMVLuc at concentrations of $20 \mu \mathrm{g} / \mathrm{mL}$ for size and $100 \mu \mathrm{g} / \mathrm{mL}$ for zeta potential measurements. The following settings were used: 10 subrun measurements per sample; viscosity for water $0.89 \mathrm{cP}$; beam mode $F(\mathrm{Ka})=1.50$ (Smoluchowsky); temperature $25{ }^{\circ} \mathrm{C}$.

Coupling of X-Rhodamine to PEI and Fluorescence Resonance Energy Transfer (FRET) Measurements. Five hundred microliters (5 $\mathrm{mg}$ ) of PEI was mixed with a 10-fold molar excess of 5(6)-carboxy$\mathrm{X}$-rhodamine $N$-succinimidyl ester, which was diluted in $100 \mu \mathrm{L}$ of DMSO. After shaking for $2.5 \mathrm{~h}$ at room temperature, the mixture was purified on a Sephadex PD-10 column using double-distilled water as elution reagent. The PEI content was determined via $\mathrm{CuSO}_{4}$ assay according to Ungaro et al. ${ }^{18}$ Complexes for FRET measurements were prepared using X-rhodamine-PEI and INS-FITC instead of PEI and INS as described above. After incubation, the fluorescence spectrum $(500-700 \mathrm{~nm})$ of the sample was measured using a Cary eclipse fluorescence spectrophotometer at an excitation wavelength of $488 \mathrm{~nm}$. As a reference, pCMVLuc complexes with either PEI/INS-FITC or $\mathrm{X}$-rhodamine-PEI/INS were used.

To investigate the effect of solvents on ternary complex formation and stability, complexes were prepared in either PBS or MEM using X-rhodamine-PEI, pCMVLuc, and insulin-FITC. After incubation, the fluorescence spectrum was measured as above. In comparison, complexes prepared in water were mixed with either PBS or MEM and measured for stability analysis.

Negative Staining of PEI-pCMVLuc and PEI-pCMVLuc-INS Complexes. For electron microscope analysis, PEI-pCMVLuc and PEI-pCMVLuc-INS complexes with concentrations of $0-25 \mu \mathrm{g}$ INS/ $\mu \mathrm{g}$ pDNA were prepared freshly as described above. Samples were incubated at room temperature prior to the preparation of electron microscopy specimens. To this end, the samples were filled into a $\sim 25$ $\mu \mathrm{L}$ well of a black polyoximethylen block. Particles were adsorbed for $90 \mathrm{~s}$ onto a piece of carbon film about $3 \mathrm{~mm} \times 3 \mathrm{~mm}$ in size, which had been previously evaporated onto freshly cleaved mica prior to usage. Subsequently, the carbon film with adsorbed particles was transferred to a second well filled with $2 \%$ uranyl formate dissolved in water for 2 min and was picked up from the staining solution by putting an electron microscopy grid covered with a holey carbon film on top of the floating carbon film. ${ }^{19}$ After blotting the excess liquid, the grid was dried at room temperature. Specimens were imaged using a Philips 
CM200 FEG electron microscope (Philips/FEI, Eindhoven, The Netherlands) operated at $160 \mathrm{kV}$ and a magnification of $70000 \times$. Images were taken at room temperature on a $4 \mathrm{k} \times 4 \mathrm{k}$ charge-coupled device (CCD) camera (TVIPS, Gauting, Germany) with 2-fold pixel coarsening. Particle dimensions were determined using the imaging software EMMENU of TVIPS.

Native Cryo Preparation of Vitrified Specimens. Samples of the PEI-pCMVLuc and PEI-pCMVLuc-INS complexes in water that were prepared for negative staining electron microscopy were also subjected to vitrification for native cryo electron microscopy. Five microliters of the samples was pipetted onto an electron microscopy grid covered with a holey carbon film, which was glow-discharged prior to usage. After briefly blotting the excess liquid, the electron microscopy grid was rapidly plunged into liquid ethane for vitrification and stored in liquid nitrogen until electron microscopy analysis. ${ }^{20}$ Images at a magnification of $70000 \times$ were taken at liquid nitrogen conditions using a Philips CM200 FEG electron microscope equipped with a Gatan cryoholder (Gatan GmbH, Munich, Germany). For electron microscopy analysis, a $4 \mathrm{k} \times 4 \mathrm{k}$ CCD camera (TVIPS) was used, and particle sizes were determined using the EMMENU software.

DNA Retardation Assay. Complexes used for transfection studies corresponding to $100 \mathrm{ng}$ of pCMVLuc were diluted in $5 \mu \mathrm{L}$ of doubledistilled water. Each sample was analyzed in duplicates. One of each of the samples was treated with $10 \mu \mathrm{g}$ of heparan sulfate (SigmaAldrich, Schnelldorf, Germany). After 45 min of incubation, samples were mixed with $2 \mu \mathrm{L}$ of loading buffer $(0.25 \%$ bromphenol blue, $0.25 \%$ xylene cyanol FF, $30 \%$ glycerol in water), loaded into individual wells of a $0.8 \%$ agarose gel, and separated at $120 \mathrm{~V}$ for $1.5 \mathrm{~h}$ by electrophoresis. Staining of the gel was performed with ethidium bromide, and bands corresponding to DNA were visualized under UV light.

PEI-pCMVLuc complexes as described for transfection studies were also prepared additionally by replacing INS with INS-FITC. These were used to visualize the binding of INS to PEI-pCMVLuc complexes by agarose gel electrophoresis. Subsequent to the formation of ternary complexes, agarose gel electrophoresis was performed as described above. No staining with ethidium bromide was performed so as to enable us to directly visualize the FITC fluorescence.

In Vitro Transfection Studies. For in vitro transfection experiments, A549 and BEAS-2B cells were seeded in 24-well plates (TPP, Trasadingen, Switzerland) $24 \mathrm{~h}$ prior to transfection with a density of 100000 cells per well. According to previous studies in our group, ${ }^{8}$ gene transfer complexes corresponding to $1 \mu \mathrm{g}$ of pCMVLuc/pEGFP$\mathrm{N} 1$ per well were pipetted onto the cells in serum-free medium. After $4 \mathrm{~h}$ of incubation, the transfection medium was replaced with MEM including 10\% FCS supplemented with antibiotics (1.0\% penicillin/ streptomycin and $0.5 \%$ gentamycin, Invitrogen). Reporter gene activity was measured $24 \mathrm{~h}$ post-transfection. Luciferase activity was measured in relative light units (RLU) using a Wallac Victor ${ }^{2} 1420$ Multilabel Counter (PerkinElmer, Boston, MA) according to Huth et al. ${ }^{21}$ Luciferase values were normalized to total cell protein using BioRad Protein Assay (BioRad, Munich, Germany) and bovine serum albumin as protein standard. For EGFP measurements, cells were subjected to FACS analysis. Five thousand cells were counted per sample.

Cell Viability Assay. Cytotoxicity of ternary PEI-pCMVLuc-INS complexes was evaluated on A549 cells with an N/P ratio of 8. Cells were seeded $24 \mathrm{~h}$ prior to the experiment with a density of 25000 cells per well into 96 -well plates. Complexes were generated according to transfection experiments and added to the cells containing a total amount of $0.5 \mu \mathrm{g}$ of pCMVLuc per well. After $4 \mathrm{~h}$, the transfection mixture was replaced with FCS-supplemented medium, and after another $24 \mathrm{~h}$, cell viability was measured using an ATPlite luminescence detection assay system (PerkinElmer) according to the manufacturer's instructions. Untreated cells were used as a reference by setting the corresponding luminescence to $100 \%$ viable cells.

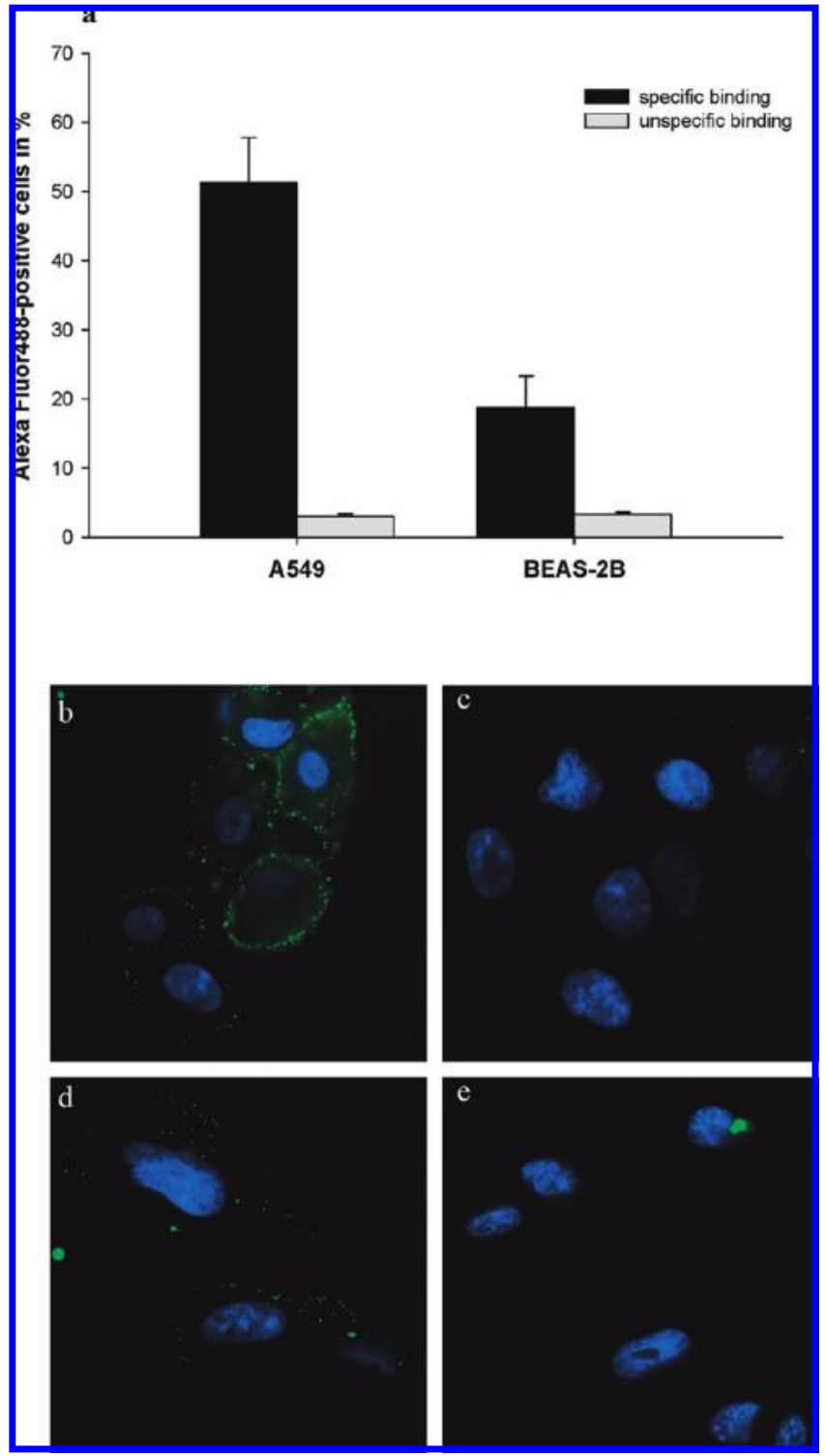

Figure 2. Distribution of INS receptors on alveolar (A549) and bronchial (BEAS-2B) epithelial cells. Incubation of antibodies was performed as described in the Experimental Section. FACS measurements on A549 and BEAS-2B cells (a). Data are given as mean \pm $\operatorname{SD}(n=3)$. Fluorescence microscopy after incubation of primary and secondary antibody on A549 (b) and BEAS-2B (d) cells. In addition, images of control experiments (only secondary antibody) were taken for A549 (c) and BEAS-2B cells (e).

Statistical Analysis. Results are reported as mean values \pm standard deviation (SD). Statistically significant differences were evaluated by a nonpaired student's $t$ test. Probabilities of $p \leq 0.05$ were considered as significant.

\section{Results}

Investigation of the INS Receptor on Lung Epithelial Cells. INS receptors on the surface of alveolar (A549) and bronchial epithelial (BEAS-2B) cells were analyzed by flow cytometry. More than 50\% of A549 but only 19\% of BEAS2B cells expressed INS receptors (Figure 2a). Unspecific binding was lower than $4 \%$ in both cell lines. These findings were further confirmed by fluorescence microscopy. On A549 cells, intense fluorescence signals were observed that were associated with cellular membranes as evident by the punctate pattern of staining (Figure 2b), whereas no signal could be detected in the control 
Table 1. Physical Characteristics of PEI-pCMVLuc and $\mathrm{PEI}-\mathrm{pCMVLuc}-\mathrm{INS}$ Complexes with Various Amounts of INS and an N/P Ratio of 8 (Complexes that were Not Measurable Due to Aggregation are Denoted with an Asterisk)

\begin{tabular}{cccc}
\hline $\begin{array}{c}\text { amount of insulin } \\
(\mu \mathrm{g} / \mu \mathrm{g} \text { pCMVLuc })\end{array}$ & size $(\mathrm{nm})$ & $\mathrm{PI}$ & $\begin{array}{c}\text { zeta potential } \\
(\mathrm{mV})\end{array}$ \\
\hline 0 & $43 \pm 1$ & 0.228 & $35 \pm 3$ \\
1 & $48 \pm 1$ & 0.235 & $37 \pm 3$ \\
2 & $152 \pm 1$ & 0.281 & $23 \pm 3$ \\
3 & $180 \pm 2$ & 0.273 & $19 \pm 2$ \\
4 & $230 \pm 4$ & 0.296 & $14 \pm 5$ \\
5 & $283 \pm 2$ & 0.246 & $15 \pm 4$ \\
10 & $*$ & $*$ & $-7 \pm 3$ \\
\hline
\end{tabular}

samples (only secondary antibody) (Figure 2c). A similar pattern could also be observed on BEAS-2B cells, but at a significantly lower fluorescence signal frequency and intensity (Figure 2d, e). These observations demonstrate that the expression of INS receptors is higher in alveolar epithelial than in bronchial epithelial cells.

Biophysical Parameters of PEI-pCMVLuc and PEIpCMVLuc-INS Particles. Particle size and zeta potential of surface-adsorbed PEI-pCMVLuc-INS complexes with an N/P of 8 were dependent on the amount of INS (Table 1). The hydrodynamic diameter of PEI-pCMVLuc polyplexes as determined by dynamic light scattering was $43 \pm 1 \mathrm{~nm}$ and gradually increased with increasing amounts of INS. With 5 $\mu \mathrm{g}$ of INS/ $\mu \mathrm{g}$ pDNA, complex size was close to $300 \mathrm{~nm}$, and with $10 \mu \mathrm{g}$ of INS/ $\mu \mathrm{g}$ pDNA, aggregation occurred. At the same time, addition of INS resulted in reduction of the zeta potential. At $10 \mu \mathrm{g}$ of INS/ $\mu \mathrm{g}$ pDNA, complexes exhibited negative zeta potential values. These results suggest successful coating of the particle surface with INS, which is able to shield the positive charge of PEI-pDNA complexes. In addition to dynamic light scattering measurements, PEI-pCMVLuc and PEI-pCMVLucINS complexes formed in water were also analyzed by electron microscopy to reveal their size, dispersity, and morphology. To this end, we analyzed the identical samples both by negative staining electron microscopy (Figure 3a, c, e, g) and native electron cryomicroscopy (Figure 3b,d,f,h). Indeed, similar results were obtained using both sample preparation and imaging procedures: no relevant differences were seen in the overall shape, appearance, dispersity/aggregation behavior, and size independent of the electron microscopy approach used. PEI-pCMVLuc complexes showed a homogeneous population of nearly globular particles with maximum dimensions of about 40-50 nm (Figure 3a for negative staining electron microscopy and Figure $3 \mathrm{~b}$ for native electron cryomicroscopy), while larger particles $(>100 \mathrm{~nm}$ ) were only occasionally seen accounting for about $5 \%$ of the particles. Overall, PEI-pCMVLuc complexes adopted a densely packed, compact appearance. Adding of INS to a concentration of $5 \mu \mathrm{g} / \mu \mathrm{g}$ of plasmid DNA resulted in an increase of particle dimensions up to about 200-300 nm, and the monodisperse appearance was still maintained (Figure $3 \mathrm{c}$, d). Likewise, the densely packed, compact morphology was preserved; however, PEI-pCMVLuc-INS complexes at this INS concentration also revealed polypoid features. In addition, a second smaller subpopulation of globular, compact particles with maximum dimensions of about $50 \mathrm{~nm}$ were also observed that showed very similar structural features as compared to the untreated PEI-pCMVLuc particles (i.e., no addition of INS) seen in Figure 3a, b. In contrast, insulin amounts of $15 \mu \mathrm{g} / \mu \mathrm{g}$ pDNA resulted in severe particle aggregation (Figure $3 \mathrm{e}, \mathrm{f}$ ). Thereby, the particles adopted a network-type appearance with maximum dimensions of more than $700 \mathrm{~nm}$. A further increase

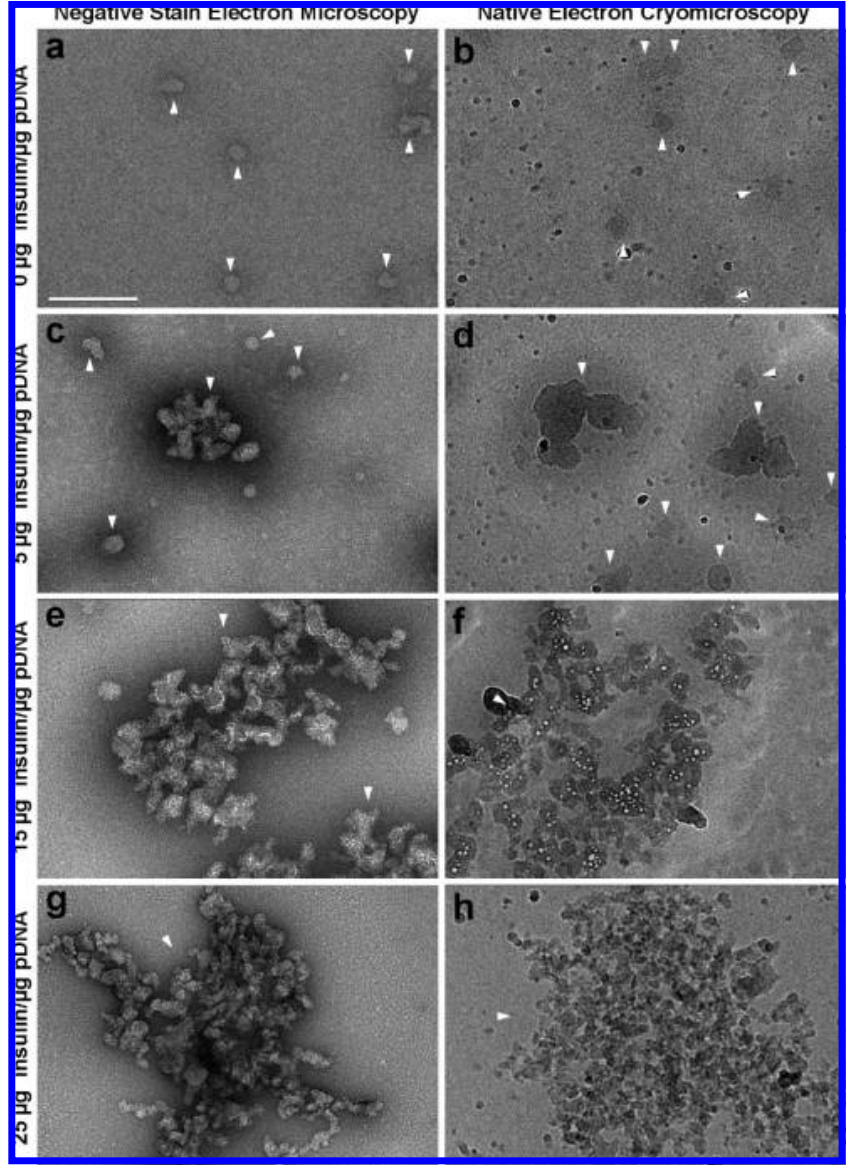

Figure 3. Electron microscopic analysis of PEI-pCMVLuc $(a, b)$ and PEI-pCMVLuc-INS complexes prepared at concentrations of 5 (c, d), $15(\mathrm{e}, \mathrm{f})$, or $25(\mathrm{~g}, \mathrm{~h}) \mu \mathrm{g}$ of INS/ $\mu \mathrm{g}$ plasmid DNA. Complexes were visualized using the negative staining (left images) or native cryo preparation (right images) method. Individual particles are marked with arrowheads. The scale bar corresponds to $200 \mathrm{~nm}$ on the specimen level.

of the INS concentration to $25 \mu \mathrm{g} / \mu \mathrm{g}$ pDNA also revealed severe particle aggregation similar to the PEI-pDNA-INS complexes at $15 \mu \mathrm{g} / \mu \mathrm{g}$ pDNA (Figure $3 \mathrm{~g}, \mathrm{~h}$ ).

Investigation of Surface-Adsorbed INS on PEI-pCMVLuc Gene Vectors by FRET Measurements and Agarose Gel Electrophoresis. Surface adsorption of INS on PEI-pCMVLuc gene vector particles was further confirmed using gene vector particles containing PEI labeled with X-rhodamine (energy acceptor) and INS labeled with fluorescein (energy donor) through fluorescence resonance energy transfer (FRET). As shown in Figure 4a, addition of INS-FITC to X-rhodamine-PEIpCMVLuc complexes led to an increase of X-rhodamine emission and to a decrease of FITC emission. This observation indicates that INS binds to the surface of PEI-pCMVLuc complexes because energy transfer only occurs between fluorophores in close proximity (typically $<6 \mathrm{~nm}$ ). ${ }^{22}$ FRET experiments were also performed following the addition of physiological solutions such as PBS (Figure 4b) or serum-free media (data not shown) to PEI-pCMVLuc-INS complexes prepared in water. Though there was dissociation of INS-FITC from the complexes upon addition of physiological solutions (as evidenced by the increase in the FITC signal), the FRET effect was still visible, albeit reduced, thus concluding that some amount of insulin was still adsorbed to PEI-pCMVLuc complexes. This observation was further confirmed by gel electrophoresis. Though there was dissociation of INS from 

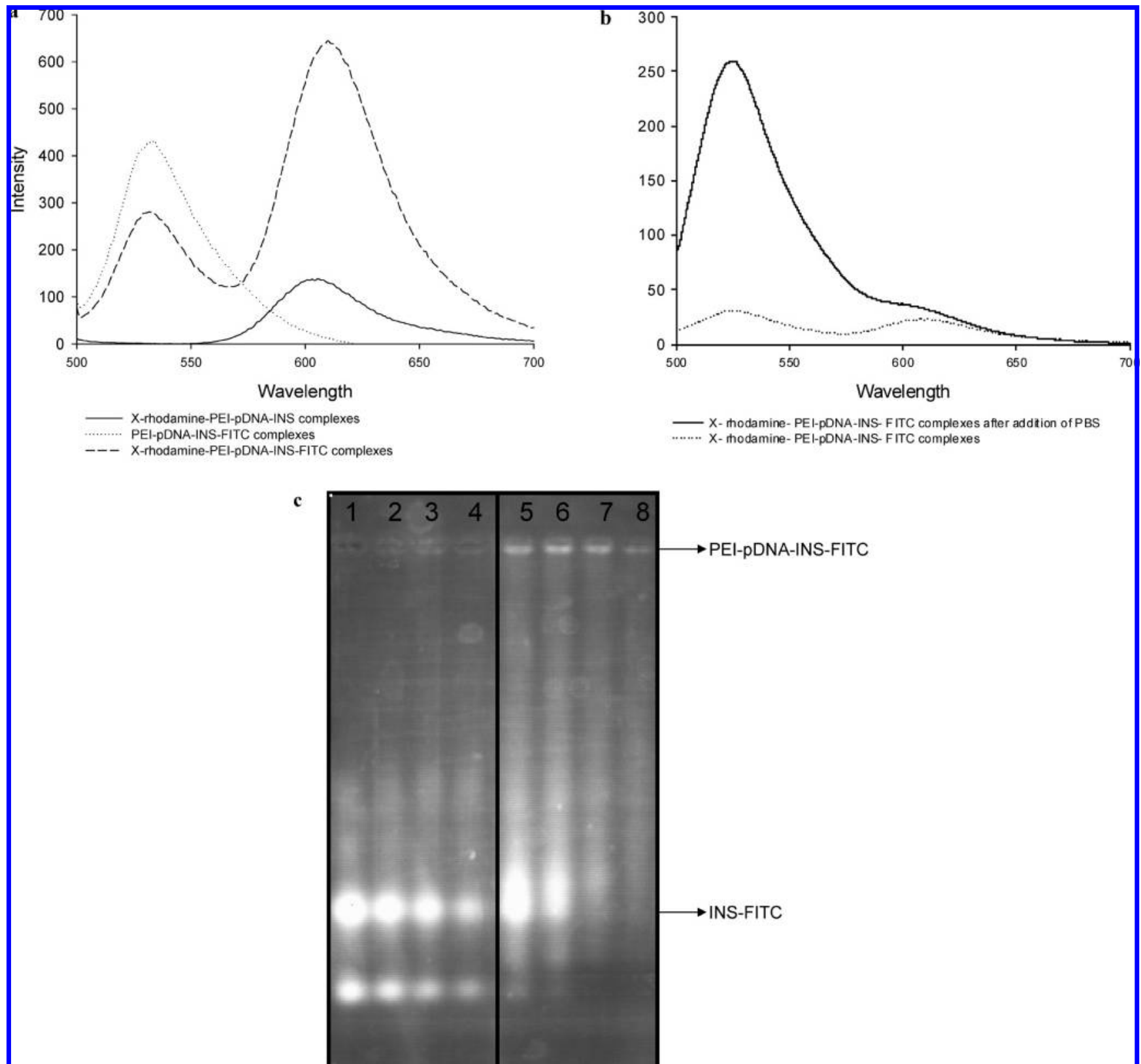

Figure 4. Surface adsorption of INS on PEI-pCMVLuc gene vector particles using gene vector particles consisting of PEI labeled with X-rhodamine (energy acceptor) and INS labeled with fluorescein (energy donor) through fluorescence resonance energy transfer (FRET). Addition of INSFITC to X-rhodamine-PEI-pCMVLuc complexes led to an increase of X-rhodamine emission and to a decrease of FITC emission (a). Effect of solvent on ternary complex formation. Addition of PBS to complexes prepared in water results in dissociation of some FITC-INS from PEI-pCMVLuc complexes. The FRET effect is still visible (b). Visualization of PEI-pCMVLuc-INS complexes by agarose gel electrophoresis. PEI-pCMVLuc-INS complexes were prepared using different amounts of INS $(2-5 \mu \mathrm{g})$ per microgram of plasmid DNA. Complexes containing $100 \mathrm{ng}$ of DNA were loaded per well. Lanes 1-4: 500, 400, 300, and $200 \mathrm{ng}$ of INS-FITC, respectively. Lanes 5-8: complexes prepared using $5,4,3$, and $2 \mu \mathrm{g}$ of INS-FITC/ $\mu \mathrm{g}$ DNA, respectively (c).

PEI-pCMVLuc-INS ternary complexes, upon addition of solutions with higher ionic strength compared to water, surface adsorption of INS-FITC to PEI-pCMVLuc complexes could nonetheless be visualized (Figure 4c). This further supports the FRET data that, even though there is dissociation of INS from PEI-pCMVLuc upon addition of physiological solutions, some amount of INS remains attached to the surface.

DNA Retardation Assay by Agarose Gel Electrophoresis. Gel retardation assay did not show any differences between PEI-pCMVLuc and PEI-pCMVLuc-INS gene vectors. Independent from the amount of INS, no pDNA could be detected in the gel, which indicates complete pDNA condensation (Figure 5). After treatment with heparan sulfate, pDNA was released from the complexes for each of the samples. These observations suggest that adsorption of INS, to the surface of PEI-pCMVLuc complexes, does not result in any weakening of the electrostatic interaction between the positively charged PEI with the negatively charged pDNA.

In Vitro Transfection Studies. In vitro transfection efficiency of PEI-pDNA and PEI-pDNA-INS complexes with an N/P of 8 was evaluated on A549 and BEAS-2B cells. Results are summarized in Figure 6. On A549 cells, INS coating of PEI-pCMVLuc complexes resulted in an increase of gene expression with increasing amounts of INS. At optimized PEI-pCMVLuc-INS ratio (5 $\mu \mathrm{g}$ INS/ $\mu \mathrm{g}$ pDNA), gene expression was significantly 16-fold higher compared to nontargeted 


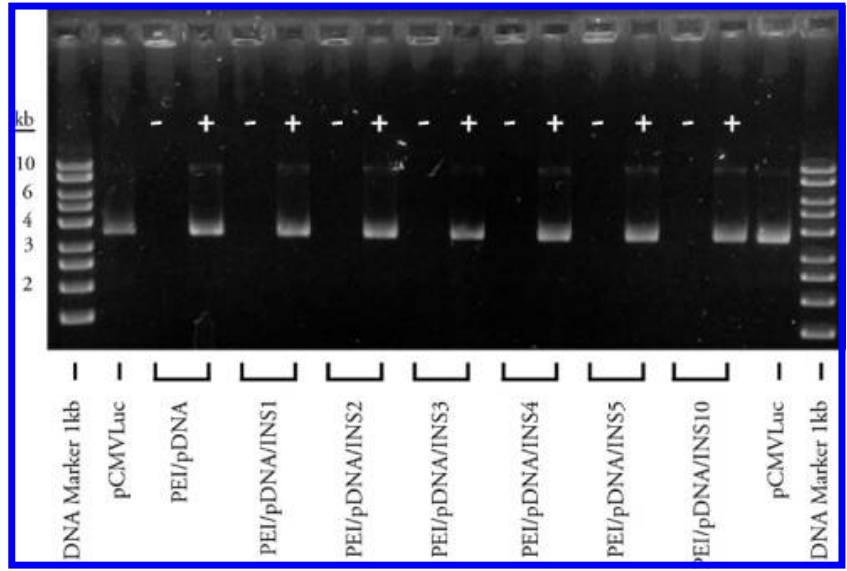

Figure 5. Gel retardation assay comparing pDNA condensation behavior of PEI-pCMVLuc with PEI-pCMVLuc-INS complexes. Combinations were incubated without $(-)$ and with $(+)$ heparan sulfate, resolved on agarose gel, stained with ethidium bromide, and visualized under UV light. Plasmid pCMVLuc was used as a control.

PEI-pCMVLuc complexes. No increase in gene expression from PEI-pCMVLuc-INS compared to PEI-pCMVLuc gene vectors was observed on BEAS-2B cells (Figure 6a).

Competitive inhibition experiments with excess of free INS were performed to show that this increase in gene expression is insulin receptor specific. A549 cells were incubated with varying concentration of free INS and PEI-pCMVLuc-INS5 complexes, which showed highest increase in luciferase expression. PEI-pCMVLuc complexes were used as a control. As shown in Figure 6b, increased gene expression observed with PEI-pCMVLuc-INS5 could be reduced to levels comparable to PEI-pCMVLuc, by addition of as little as $0.001 \mathrm{mg} / \mathrm{mL}$ free insulin. Increasing the concentrations of free insulin further did not result in any more reduction in expression.

We next aimed to investigate if the observed INS specific increased transfection efficiency was due to higher number of transfected cells or better expression of the transgene when delivered via targeting the insulin receptor. For this, cells were transfected with an EGFP expression plasmid, and FACS measurements were made $24 \mathrm{~h}$ post gene delivery. Similar to our luciferase data, effect of INS was observed only in A549 cells. In these cells, both the number of EGFP positive cells (19 $\pm 1 \%$ with PEI-pEGFP compared to $32 \pm 6 \%$ with PEI-pEGFP-INS5 complexes) and mean fluorescence intensity $(127 \pm 24$ with PEI-pEGFP compared to $330 \pm 56$ with PEI-pEGFP-INS5 complexes) were significantly increased ( $p$ $\leq 0.05)$. Thus transfection with PEI-pDNA-INS5 results in both higher number of transfected cells and increased expression of the transgene per cell.

In order to evaluate whether enhanced gene expression is a result of proliferative effects of INS on the cells, we transfected A549 cells with PEI-pCMVLuc complexes prepared at an N/P ratio of 8 in the presence of various concentrations of free INS in the transfection medium, ranging from 0.001 to $0.25 \mathrm{mg}$ / $\mathrm{mL}$. With $0.25 \mathrm{mg} / \mathrm{mL}$, the total amount of INS per well was 12.5-fold higher compared to the highest amount of coated INS on PEI-pDNA-INS complexes. Co-incubation did not result in any significant changes of luciferase expression (Figure 6c). As a result, we conclude that insulin in the concentration range we used did not influence cell proliferation, which may affect transfection efficiency. These findings demonstrate that coating of PEI-pDNA complexes with optimal amounts of INS could improve gene delivery and expression from PEI-pDNA complexes on alveolar epithelial but not on bronchial epithelial cells.

Cytotoxicity. Cell viability after application of gene vectors was measured using an ATP assay, which indicates metabolic cell activity. PEI-pCMVLuc complexes showed significant cell death compared to untreated cells (Figure 7). In contrast, no cytotoxicity was observed with each of the PEI-pCMVLuc-INS complexes, even when the lowest ratio of $1 \mu \mathrm{g}$ INS/ $\mu \mathrm{g}$ pDNA was used. Therefore, we suggest that surface adsorption of INS prevents cytotoxic effects of PEI-pDNA complexes.

\section{Discussion}

In this study, we investigated whether noncovalent binding of INS to the surface of PEI-pDNA complexes could improve nonviral gene expression in human lung epithelial cells. The principal finding of our study is that transgene delivery and expression increased on alveolar epithelial cells, which express INS receptors at high density, but not on bronchial epithelial cells, which express INS receptors only at low levels. In addition, INS coating significantly reduced cytotoxicity of PEI-pDNA complexes.

We demonstrated by antibody staining that INS receptors are predominantly expressed with a high density on alveolar epithelial cells but only with low density on bronchial epithelial cells. This was shown by FACS analysis and confirmed by fluorescence microscopy. In this context, INS receptors on alveolar epithelial cells have been previously described. ${ }^{13}$ Thus, we speculated that INS receptor might be a promising candidate for enhancing gene delivery and expression specifically in alveolar epithelial cells with high density of INS receptors.

In this study, we used the cationic polymer PEI $25 \mathrm{kDa}$ as gene transfer agent, which has been previously shown to result in higher gene transfer efficiency in the lung than the more frequently used linear PEI $22 \mathrm{kDa} .{ }^{23}$ Insulin was adsorbed on the surface of preformed PEI-pDNA complexes, resulting in targeted ternary PEI-pDNA-INS polyplexes. Electrostatic adsorption was favored over covalent binding in order to avoid alterations of insulin's structure, which could occur during chemical coupling procedures and cause loss of INS activity and thus binding to INS receptors. In general, two different ways of forming ternary complexes are conceivable. The first possibility is to mix the INS with PEI prior to complexation with pDNA ("peptide first" method). Alternatively, PEI-pDNA complexes are first formed, and subsequently, the positive surface is coated with negatively charged INS ("polymer first" method). We chose the second option to avoid masking of INS within the PEI-pDNA gene vectors and therefore preserve its accessibility for receptor binding.

Characterization of the particle size using dynamic light scattering showed that increasing the amount of INS enlarged hydrodynamic diameters. Whereas INS addition of $5 \mu \mathrm{g} / \mu \mathrm{g}$ of pDNA still led to defined particles smaller than $300 \mathrm{~nm}$, doubling the INS amount caused aggregation. These findings were further confirmed by electron microscopy. Surface coating of PEI-pCMVLuc nanoparticles with INS not only resulted in an increase of the particle size but also led to a decrease in the zeta potential to negative values. On the basis of these results, we suggest successful adsorption of INS on the particle surface. In addition, we could demonstrate with a gel retardation assay that pDNA was effectively complexed in all combinations irrespective of the INS amount. Thus surface adsorption of INS does not affect the electrostatic binding of positively charged PEI to negatively charged DNA. 


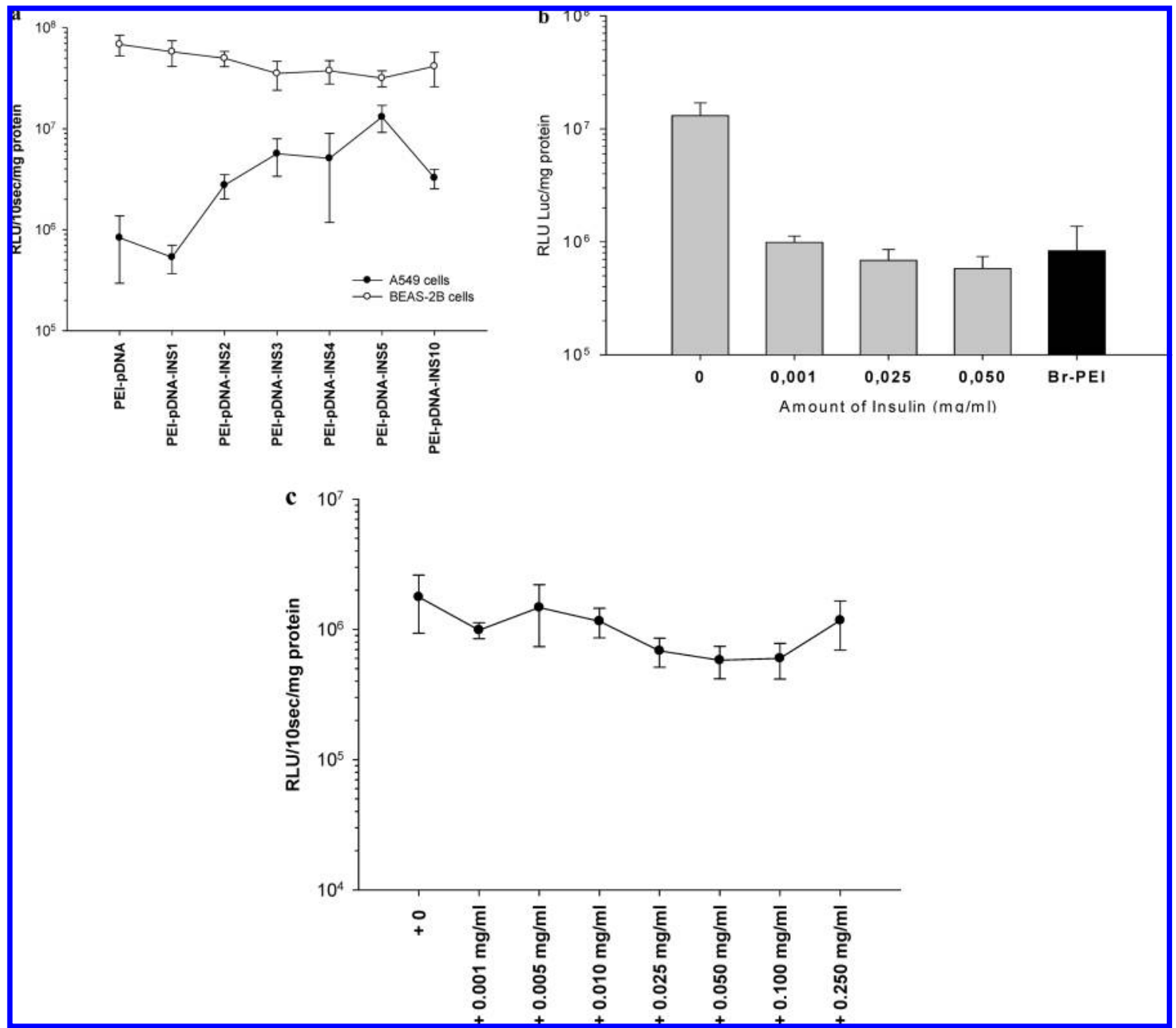

Figure 6. Transfection efficiencies of PEI-pCMVLuc and PEI-pCMVLuc-INS complexes at an N/P ratio of 8 . A549 and BEAS-2B cells were transfected with polyplexes containing different amounts of insulin (a). The increase in gene expression with PEI-pCMVLuc-INS complexes in alveolar cells could be inhibited by excess free insulin to levels comparable to PEI-pCMVLuc complexes (b). To exclude false positive results due to proliferative effects of insulin, transfection efficiency of PEI-pCMVLuc complexes was investigated on A549 cells in the presence of various concentrations of insulin in the medium (c). Luciferase gene expression was measured as relative light units (RLU) luminescence during $10 \mathrm{~s} / \mathrm{mg}$ of cellular protein. The data are given as mean $\pm \mathrm{SD}(n=4)$. Statistically significant differences to PEI-pCMVLuc transfection efficiencies are denoted with an asterisk $(p \leq 0.05)$.

Adsorption of INS on PEI-pCMVLuc gene vector particles was further confirmed with FRET. PEI was labeled with X-rhodamine (energy acceptor) and INS with fluorescein (energy donor). Coupling of INS-FITC to X-rhodamine-PEI-pCMVLuc complexes in water resulted in clear FRET effect, thus confirming the adsorption of INS onto PEI-CMVLuc complexes since transfer occurs between fluorophores only in close proximity $(<6 \mathrm{~nm}){ }^{22}$ Addition of physiological solutions such as PBS and serum-free media to PEI-pCMVLuc-INS complexes prepared in water revealed dissociation of some amounts of INS-FITC from the ternary complexes. However, significant amounts of INS still remained adsorbed to PEI-pCMVLuc-INS complexes in PBS and serum-free media, resulting in reduced albeit detectable FRET signals. Similar to observations made with FRET, dissociation of INS from PEI-pCMVLuc-INS, upon loading onto an agarose gel prepared in TAE buffer $(40 \mathrm{mM}$ Tris, $20 \mathrm{mM}$ acetate, and $10 \mathrm{mM}$ EDTA), could be observed. Nonetheless, significant amounts of INS-FITC remained adsorbed to the complexes. This observation further supports our FRET data that solutions of high ionic strength result in partial dissociation of INS from PEI-pCMVLuc-INS complexes. However, the amount of INS that remains adsorbed to the complexes is sufficient to result in higher gene expression seen in transient transfection studies and also to reduce the cytotoxicity of PEI-pDNA complexes.

In transfection experiments, coating with INS resulted in enhanced gene delivery and expression in alveolar but not in bronchial epithelial cells. Using $5 \mu \mathrm{g}$ of INS/ $\mu \mathrm{g}$ of pCMVLuc led to a maximum of gene expression with a significant 16 -fold increase compared to unmodified PEI-pCMVLuc complexes. The increased gene expression levels with PEI-pCMVLuc-INS5 could be reduced to those observed for PEI-pCMVLuc complexes by excess of free insulin. Though PEI-pCMVLucINS complexes were not purified, to remove unattached free insulin, before transfecting the cells, competitive inhibition experiments with excess insulin suggest that, under the conditions used to prepare the complexes, there is not much free insulin in the mixture. Further experiments with PEI-pEGFP and PEI-pEGFP-INS5 provided evidence that both the number of transfected cells and transgene expression per cell were significantly enhanced by INS adsorption to the complexes. Similar to observations made in luciferase assays, this increase was restricted to A549 cells. 


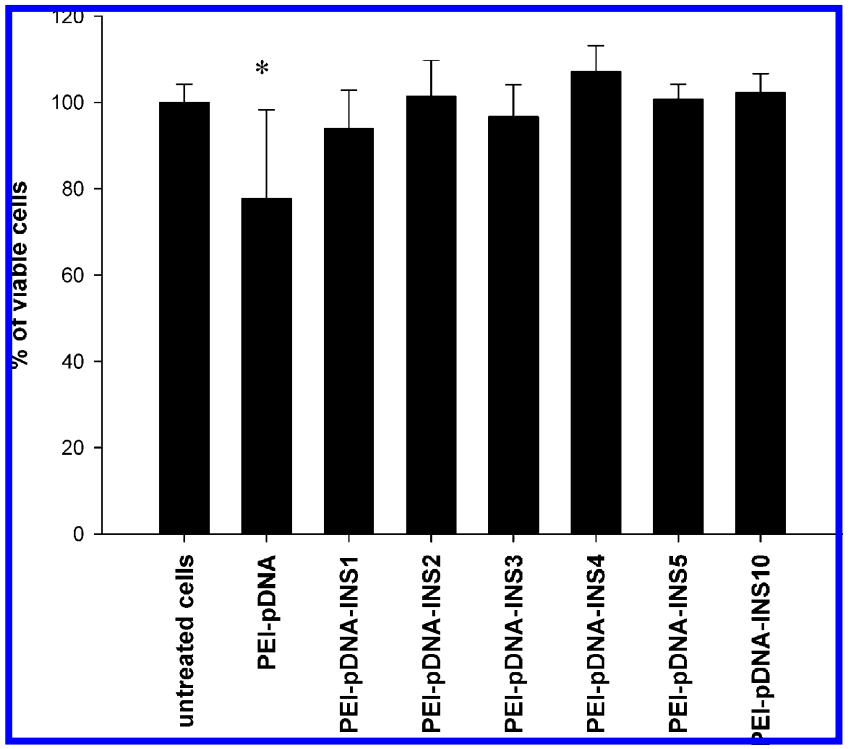

Figure 7. Cell viability of $A 549$ cells after treatment with PEI-pCMVLuc or PEI-pCMVLuc-INS complexes at an N/P ratio of 8 . Measurements of the ATP luminescence were performed $24 \mathrm{~h}$ post-transfection. Values of untreated cells were taken to be $100 \%$ for calculating purposes. The data are given as mean $\pm \mathrm{SD}(n=8)$. Statistically significant differences from complexes to untreated cells are denoted with an asterisk $(p \leq 0.05)$.

To exclude false positive results due to proliferative effects of INS on the cells, we examined gene expression on A549 cells with PEI-pCMVLuc complexes in the presence of free INS. No significant change of luciferase expression could be observed. Hence, we conclude that cell proliferation plays no major role in our studies. Adsorption of free INS to PEI-pCMVLuc complexes and the subsequent increase in expression (as seen with PEI-pCMVLuc-INS complexes) was not visible in this experiment. This observation is explained by the FRET data which demonstrate that the ternary complexation of INS to PEI-pDNA is most effective when carried out in water. When the complexes are made in PBS or serum-free medium, hardly any energy transfer (reflecting lack of adsorption) could be observed (data not shown).

However, it is surprising that INS adsorption on PEI-pDNA complexes did not result in an increase of gene delivery or expression on bronchial epithelial cells, although they were found to express INS receptors. We suggest that this discrepancy may be a result of the low INS receptor density found on bronchial epithelial cells compared to alveolar epithelial cells, which was observed by fluorescence microscopy. It therefore seems to be likely that a threshold of INS receptor density on the cellular surface is necessary for successful INS-mediated gene delivery. Another contributor to the observed differences between the two cell types could be the INS receptor itself, which may be coupled to different downstream signaling pathways in the two cell types.

Cytotoxicity assays revealed that INS coating reduced the cytotoxicity of PEI-pCMVLuc complexes. The high toxicity of unmodified PEI-pCMVLuc complexes may primarily be driven by the surface charge. Observations made by Hong et al. demonstrated that cytotoxicity is closely related to the density of charged groups of polymer backbones. ${ }^{24}$ Therefore, coating of the PEI-pCMVLuc gene vector surface with INS exhibits a straightforward strategy, which reduces cytotoxicity while improving transfection efficiency and cell specificity at the same time. Additional experiments need to be performed before the in vivo application of such INS-modified polyplexes. For example, it needs to be investigated if the ternary complexes could survive the nebulizing process for lung gene delivery. In this context, it remains to be examined if the noncovalent INS attachment is sufficient or if it needs to be bioreversibly crosslinked upon surface adsorption. Moreover, the effect of extracellular matrix in the lung (like bronchio-alveolar fluid) on the stability of PEI-pDNA-INS complexes needs to be addressed in detail. This aspect is of critical value since our FRET data show partial dissociation of INS from the complexes upon addition of physiological media/buffers.

\section{Conclusion}

In this study, we investigated ternary complexes formed via electrostatic interactions between branched PEI, pDNA, and human INS for their ability to improve nonviral gene expression in lung epithelial cells. We demonstrated a higher number of INS receptors on alveolar epithelial cells compared to bronchial epithelial cells. In agreement with this observation, ternary PEI-pDNA-INS complexes resulted in an up to 16-fold increase of gene expression compared to PEI-pDNA complexes on A549 cells. In contrast, no significant difference was obtained with bronchial epithelial cells. Particle size, charge, and FRET measurements demonstrated the formation of ternary complexes. In addition to increase in gene expression, adsorption of INS decreased cytotoxicity of PEI-pDNA complexes. We therefore suggest that INS represents a promising targeting ligand for receptor-mediated gene delivery to the alveolar epithelium.

Acknowledgment. This work was supported by the BMBF BioFuture program (FKZ 0311898 and 0311899) to C.R. and H.S., respectively, and LMUexcellent (Investitionsfonds).

\section{References and Notes}

(1) Themis, M.; Waddington, S. N.; Schmidt, M.; von Kalle, C.; Wang, Y.; Al-Allaf, F.; Gregory, L. G.; Nivsarkar, M.; Holder, M. V.; Buckley, S. M.; Dighe, N.; Ruthe, A. T.; Mistry, A.; Bigger, B.; Rahim, A.; Nguyen, T. H.; Trono, D.; Thrasher, A. J.; Coutelle, C. Oncogenesis following delivery of a nonprimate lentiviral gene therapy vector to fetal and neonatal mice. Mol. Ther. 2005, 12 (4), 763-771.

(2) Boussif, O.; Lezoualc'h, F.; Zanta, M. A.; Mergny, M. D.; Scherman, D.; Demeneix, B.; Behr, J. P. A versatile vector for gene and oligonucleotide transfer into cells in culture and in vivo: polyethylenimine. Proc. Natl. Acad. Sci. U.S.A. 1995, 92 (16), 7297-7301.

(3) Lemkine, G. F.; Demeneix, B. A. Polyethylenimines for in vivo gene delivery. Curr. Opin. Mol. Ther. 2001, 3 (2), 178-182.

(4) Kopatz, I.; Remy, J. S.; Behr, J. P. A model for non-viral gene delivery: through syndecan adhesion molecules and powered by actin. J. Gene Med. 2004, 6 (7), 769-776.

(5) Li, S.; Tan, Y.; Viroonchatapan, E.; Pitt, B. R.; Huang, L. Targeted gene delivery to pulmonary endothelium by anti-PECAM antibody. Am. J. Physiol. Lung Cell Mol. Physiol. 2000, 278 (3), L504-L511.

(6) Kunath, K.; Merdan, T.; Hegener, O.; Haberlein, H.; Kissel, T. Integrin targeting using RGD-PEI conjugates for in vitro gene transfer. J. Gene Med. 2003, 5 (7), 588-599.

(7) Kircheis, R.; Kichler, A.; Wallner, G.; Kursa, M.; Ogris, M.; Felzmann, T.; Buchberger, M.; Wagner, E. Coupling of cell-binding ligands to polyethylenimine for targeted gene delivery. Gene Ther. 1997, 4 (5), 409-418.

(8) Elfinger, M.; Maucksch, C.; Rudolph, C. Characterization of lactoferrin as a targeting ligand for nonviral gene delivery to airway epithelial cells. Biomaterials 2007, 28 (23), 3448-3455.

(9) Zanta, M. A.; Boussif, O.; Adib, A.; Behr, J. P. In vitro gene delivery to hepatocytes with galactosylated polyethylenimine. Bioconjugate Chem. 1997, 8 (6), 839-844.

(10) Weiss, S. I.; Sieverling, N.; Niclasen, M.; Maucksch, C.; Thunemann, A. F.; Mohwald, H.; Reinhardt, D.; Rosenecker, J.; Rudolph, C. Uronic acids functionalized polyethyleneimine (PEI)-polyethyleneglycol (PEG)graft-copolymers as novel synthetic gene carriers. Biomaterials 2006, 27 (10), 2302-2312. 
(11) Guo, W.; Lee, R. L. Receptor-targeted gene delivery via folateconjugated polyethylenimine. AAPS PharmSci 1999, 1 (4), E19.

(12) Blessing, T.; Kursa, M.; Holzhauser, R.; Kircheis, R.; Wagner, E. Different strategies for formation of pegylated EGF-conjugated PEI/ DNA complexes for targeted gene delivery. Bioconjugate Chem. 2001, 12 (4), 529-537.

(13) Shapiro, D. L.; Livingston, J. N.; Maniscalco, W. M.; Finkelstein, J. N. Insulin receptors and insulin effects on type II alveolar epithelial cells. Biochim. Biophvs. Acta 1986, 885 (2), 216-220.

(14) Harada, S.; Smith, R. M.; Jarett, L. Mechanisms of nuclear translocation of insulin. Cell Biochem. Biophys. 1999, 31 (3), 307-319.

(15) Shah, N.; Zhang, S.; Harada, S.; Smith, R. M.; Jarett, L. Electron microscopic visualization of insulin translocation into the cytoplasm and nuclei of intact H35 hepatoma cells using covalently linked nanogold-insulin. Endocrinology 1995, 136 (7), 2825-2835.

(16) Soler, A. P.; Thompson, K. A.; Smith, R. M.; Jarett, L. Immunological demonstration of the accumulation of insulin, but not insulin receptors, in nuclei of insulin-treated cells. Proc. Natl. Acad. Sci. U.S.A. 1989, 86 (17), 6640-6644.

(17) Zhang, Y.; Boado, R. J.; Pardridge, W. M. Marked enhancement in gene expression by targeting the human insulin receptor. $\underline{\text { J. Gene Med. }}$. 2003, 5 (2), 157-163.

(18) Ungaro, F.; De Rosa, G.; Miro, A.; Quaglia, F. Spectrophotometric determination of polyethylenimine in the presence of an oligonucle- otide for the characterization of controlled release formulations. J. Pharm. Biomed. Anal. 2003, 31 (1), 143-149.

(19) Valentine, R. C.; Shapiro, B. M.; Stadtman, E. R. Regulation of glutamine synthetase. XII. Electron microscopy of the enzyme from Escherichia coli. Biochemistrv 1968, 7 (6), 2143-2152.

(20) Adrian, M.; Dubochet, J.; Lepault, J.; McDowall, A. W. Cryo-electron microscopy of viruses. Nature 1984, 308 (5954), 32-36.

(21) Rosenecker, J.; Huth, S.; Rudolph, C. Gene therapy for cystic fibrosis lung disease: current status and future perspectives. Curr. Opin. Mol. Ther. 2006, 8 (5), 439-445.

(22) Itaka, K.; Harada, A.; Nakamura, K.; Kawaguchi, H.; Kataoka, K. Evaluation by fluorescence resonance energy transfer of the stability of nonviral gene delivery vectors under physiological conditions. Biomacromolecules 2002, 3 (4), 841-845.

(23) Rudolph, C.; Ortiz, A.; Schillinger, U.; Jauernig, J.; Plank, C.; Rosenecker, J. Methodological optimization of polyethylenimine (PEI)based gene delivery to the lungs of mice via aerosol application. J. Gene Med. 2005, 7 (1), 59-66.

(24) Hong, S.; Leroueil, P. R.; Janus, E. K.; Peters, J. L.; Kober, M. M.; Islam, M. T.; Orr, B. G.; Baker, J. R., Jr.; Banaszak Holl, M. M. Interaction of polycationic polymers with supported lipid bilayers and cells: nanoscale hole formation and enhanced membrane permeability. Bioconjugate Chem. 2006, 17 (3), 728-734.

BM900707J 